\title{
JNK suppresses pulmonary fibroblast elastogenesis during alveolar development
}

\author{
Sheng Liư ${ }^{1}$, Harikrishnan Parameswaran², Sarah M Young ${ }^{1}$ and Brian M Varisco ${ }^{1,3,4^{*}}$
}

\begin{abstract}
Background: The formation of discrete elastin bands at the tips of secondary alveolar septa is important for normal alveolar development, but the mechanisms regulating the lung elastogenic program are incompletely understood. JNK suppress elastin synthesis in the aorta and is important in a host of developmental processes. We sought to determine whether JNK suppresses pulmonary fibroblast elastogenesis during lung development.

Methods: Alveolar size, elastin content, and mRNA of elastin-associated genes were quantitated in wild type and JNK-deficient mouse lungs, and expression profiles were validated in primary lung fibroblasts. Tropoelastin protein was quantitated by Western blot. Changes in lung JNK activity throughout development were quantitated, and pJNK was localized by confocal imaging and lineage tracing.

Results: By morphometry, alveolar diameters were increased by $7 \%$ and lung elastin content increased 2-fold in JNK-deficient mouse lungs compared to wild type. By Western blot, tropoelastin protein was increased 5-fold in JNK-deficient lungs. Postnatal day 14 (PND14) lung JNK activity was 11-fold higher and pJNK:JNK ratio 6-fold higher compared to PN 8 week lung. Lung tropoelastin, emilin-1, fibrillin-1, fibulin-5, and lysyl oxidase mRNAs inversely correlated with lung JNK activity during alveolar development. Phosphorylated JNK localized to pulmonary lipofibroblasts. PND14 JNK-deficient mouse lungs contained 7-fold more tropoelastin, 2,000-fold more emilin-1, 800-fold more fibrillin-1, and 60-fold more fibulin-5 than PND14 wild type lungs. Primarily lung fibroblasts from wild type and JNK-deficient mice showed similar differences in elastogenic mRNAs.
\end{abstract}

Conclusions: JNK suppresses fibroblast elastogenesis during the alveolar stage of lung development.

Keywords: Lung development, Elastin, c-terminal Jun kinase, Rho kinase

\section{Background}

Beginning at approximately 35 weeks gestation and continuing for several years postnatally [1], large saccular air spaces in the lung are progressively divided into smaller ones by secondary alveolar septa leading to a twentyfold increase in alveolar surface area [2]. This exponential increase in gas-exchange capacity is essential to meeting the bioenergetic needs of ex utero growth and development. Formation of a low-compliance elastin band at the leading edge of a growing secondary alveolar septal ridge likely plays critical mechano-developmental role in alveolar septation [3-6].

Elastin is necessary for structure and function of both vascular tissues and epithelial-containing organs. It is critical to

\footnotetext{
*Correspondence: brian.varisco@cchmc.org

'Cincinnati Children's Hospital Research Foundation, Cincinnati, OH, USA

${ }^{3}$ University of Cincinnati School of Medicine, Cincinnati, OH, USA

Full list of author information is available at the end of the article
}

the hysteretic properties of both arteries [7] and lung [8] and to the elasticity of other epithelial organs such as skin and intestine. In infants with impaired alveolar development [9] and in animal models of the same [10-12], elastin fiber density and organization is disrupted. Regulators of elastogenesis (formation of elastin fibers) such as transforming growth factor- $\beta$ [13], fibroblast growth factor-2 [14], epidermal growth factor [15], and insulin like growth factor-1 [16] are well described. With regards to signaling in the fibroblast, pro-elastogenic cytokines generally activate SMAD-signaling pathways and anti-elastogenic activate mitogen-activated protein kinases $1 \& 3$ (erk 2\&1) [17]. The spatial-temporal regulation of pro- and anti-elastogenic signals is likely of critical importance since the formation of discrete elastin bands is critical to both alveolar and vascular development, and elastin experiences virtually no turnover with a half live of 74 years [18] despite expression of pro-elastogenic cytokines 
throughout life [19]. In the arterial vasculature, JNK signaling has been shown to suppress elastogenesis [20], but no such role has been demonstrated in the lung. We hypothesized that activation of JNK suppresses elastogenesis during alveolar lung development contributing to the localization of elastin to alveolar septal tips. To test this hypothesis, we assessed JNK activation during lung development, localized phosphorylated JNK by confocal microscopy, quantitated alveolar development and elastin localization in JNK deficient mice, and quantitated mRNA levels of elastin-associated genes in JNK deficient mouse lungs and primary fibroblasts.

\section{Methods}

\section{Animal use}

C57BL/6J wild-type, B6.Mapk8 $8^{t m 1 F l v} / \mathrm{J} \quad\left(J N K 1^{-/-}\right), \quad$ B6.

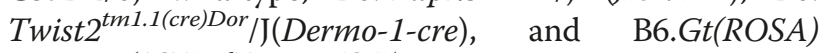

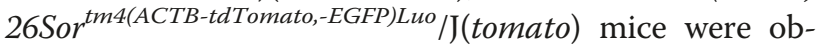
tained from Jackson Laboratories. Previously described mice with a floxed JNK1 allele $\left(M a p k 8^{t m 2 F l v} / J\right.$, referred to as $\left.J N K 1^{\text {LoxP/LoxP }}\right)$ on a B6.Mapk $9^{\text {tm1Flv }}$ Mapk $10^{\text {tm1Flv }} / \mathrm{J}$ background (hereafter referred to as $J N K 2 \& 3^{-/-}$) were utilized with permission from Roger Davis at the University of Massachusetts [21]. Since JNK3 is expressed only in the heart, testis and brain [22], single JNK2 or JNK3 knockout animals were not utilized. All animals were housed in a barrier facility with purified air and provided purified water and autoclaved food ad libitum. Animal use was approved by the Cincinnati Children's Hospital Medical Center Animal Use and Care Committee.

To localize lung JNK activity, we utilized a mesenchymal cell lineage tracing strategy with immunofluorescent staining of pJNK. Dermo1 (a.k.a. twist2) is mesenchymal transcription factor expressed as early as E15.5 [23]. The "tomato" mouse constitutively expresses a membranebound td-tomato except in the presence of cre in which recombination excises td-tomato and eGFP is expressed. Crossing Dermo1-cre mice with "tomato" mice lineage traces lung mesenchymal cells with eGFP.

\section{Genotyping}

Mice were genotyped using the primers listed in Table 1.

\section{Lung tissue processing and analysis} Tissue procurement and processing

Mice were sacrificed by intraperitoneal injection of ketamine, xylazine, and acepromazine $(100,6$, and $2 \mathrm{mg} / \mathrm{kg}$ respectively) and severing of the left renal artery. After exsanguination, the trachea was cannulated with a 22 gauge blunt tip needle and lungs isovolumetrically inflated with $4 \%$ paraformaldehyde in PBS at a pressure of $25 \mathrm{~cm} \mathrm{H}_{2} \mathrm{O}$. Lung inflation was maintained by securing a silk ligature around the trachea, and then the chest was opened. The lungs were removed and then fixed overnight at $4^{\circ} \mathrm{C}$. After fixation, the lung lobes were removed from the bronchi and dehydrated by serial passage into $70 \%$ ethanol and paraffinized. Five $\mu \mathrm{m}$ sections obtained at random angles through all five lobes.

\section{Lung elastin staining and quantification}

Lung sections were stained for elastin using the Hart's Staining method (PolyScientific) and elastin quantitated by previously published methods [24]. Using a Zeiss Axio Imager A.2 three 20X images were obtained from each lobe yielding fifteen images per mouse. Using MatLab (MatWorks) these images were then separated into four components by cluster analysis after defining a color spectrum and intensity values for dense elastin (arterial walls and elastin bands at septal tips), diffuse elastin (elastin in alveolar walls and bases), non-elastin tissue, and airspace. Pixel quantitation for each component was performed for each image and average values per animal used for statistical analysis. For quantitation, the fraction of each component of total tissue (dense elastin + diffuse elastin + non-elastin tissue) was utilized.

\section{Morphometry}

Mean alveolar diameter was calculated utilizing a published morphometric MatLab program [8,25-28], and fractional airspace was calculated using previously described methods [29].

By defining secondary alveolar crests as invaginations into distal lung airspace by tissues associated with an

Table 1 Genotyping primers used

\begin{tabular}{lll}
\hline Allele & Common forward primer & Reverse primer \\
\hline JNK1-null & CCAGGCTCTCCTCATCTTCA & Wild Type-CAGCTCATTCCTCCACTCATG \\
JNK2-null & Mutant-TCACCACATAAGGCGTCATC \\
JNK3-null & GTAGACAATCCCAGAGGTTGTGTG & Wild Type-CCAGCTCATTCCTCCACTCATG \\
& & Mutant-GGAGCCCGATAGTATCGAGTTACC \\
JNK1-floxed & Wild Type-TCCAGACTGCCTTGGGAAAA \\
\hline
\end{tabular}


elastin bundle, numbers of secondary alveolar septal crests were counted per 20X field.

\section{Immunofluorescence}

Dermo1-cre-tomato mice were sacrificed at PND5 and the lungs immunostained for pJNK using an AF647 secondary antibody. Confocal images were obtained using a Nikon AR1si inverted microscope.

\section{Tissue culture}

\section{Pulmonary fibroblast isolation}

Pulmonary fibroblasts were isolated from PND14 mice by previously described methods which yield $>85 \%$ fibroblast purity $[30,31]$. Briefly, PND14 mice were sacrificed and their lungs inflated by gently instilling dispase (BD Biosciences) via the trachea and then plugging the trachea with $1 \%$ low melting point agarose. The lungs were incubated in dispase at $25^{\circ} \mathrm{C}$ for 45 minutes and lung tissue teased from bronchi and large vessels using sterile forceps. Dispase was neutralized using DMEM with $10 \%$ FBS and the fibroblasts were allowed to adhere in a $100 \mathrm{~cm}^{2}$ tissue culture dish for 1 hour at $37^{\circ} \mathrm{C}$ and the plate rinsed with PBS. The fibroblasts were allowed to grow in DMEM with 10\% FBS and 1\% Penicillin/ Streptomycin and used at passage 3.

\section{In vitro Cre-recombinase experiments}

$J N K 2 \& 3^{-/-}$(which also has floxed JNK1 alleles) pulmonary fibroblasts were infected at $50 \%$ confluence with $10^{6}$ plaque forming units of replication-deficient adenovirus expressing GFP or Cre (Vector BioLabs). Efficacy was assessed by Taqman qPCR for JNK1 mRNA (Applied Biosystems, proprietary primers). RNA and cell culture media was collected at 48 hours.

\section{JNK activity, protein, RNA quantification JNK activity assays}

For in vivo experiments, lung homogenate c-Jun phosphorylation was quantitated to measure JNK activity. To do so, JNK was immunoprecipitated from lung homogenates using an isoform nonspecific JNK antibody (Santa Cruz) conjugated to agarose beads. Precipitated JNK was incubated with GST-labeled c-Jun (Michal Karin, University of California at San Diego) [32] and P-32 adenosine triphosphate, the kinase solution was separated on a polyacrylamide gel, transferred to a PVDF membrane, and radiolabeled c-Jun quantitated using a Storm 860 phosphorimager.

\section{Western blot}

Lungs from mice of at least two different litters were snap frozen and homogenized in RIPA buffer with protease inhibitor cocktail (Sigma) using a Qiagen TissueLyser II. Protein content was determined and the homogenates were electrophoretically separated and transferred to PVDF membrane. When not frozen, lysates were kept on ice until electrophoresis. Western blot for pJNK and JNK (both isoform non-specific, 1:200 dilution, Santa Cruz) and tropoelastin (1:2000 dilution, Elastin Products Company) was performed and chemiluminisence detected using a General Electric LAS3000.

\section{$P C R$}

Lungs from mice aged E15.5 to 8 weeks were snap frozen and then homogenized in RLT buffer using a Qiagen TissueLyser II. For primary lung fibroblasts, passage two cells were seeded at 50\% density and collected at 48 hours (with cells achieving 100\% density). Tropoelastin, emilin1, fibrillin-1, lysyl oxidase, fibulin-5, surfactant protein B, CD31, and GAPDH mRNA was quantitated by Taqman PCR (Applied Biosystems proprietary primers).

\section{Soluble elastin quantification}

Pulmonary fibroblasts were cultured for 48-hours in DMEM, 10\% FBS, 1\% penicillin/streptomycin and the media analyzed for elastin content using the Fastin kit (Biocolor, UK) per manufacturer instructions.

\section{Statistical analysis}

Statistical comparisons between groups were performed using the Student two-tail $t$-test or one-way ANOVA using the Holm-Sidak method. p-values of $<0.05$ were considered significant.

\section{Results}

JNK-deficient mice have enlargement of distal lung airspaces

The lungs of PND14 $J N K 1^{-/-}$and $J N K 2 \& 3^{-/-}$mice appeared to have larger airspaces with fewer alveoli as compared to wild type mice (Figure 1A-C). Mean alveolar diameter was increased by $2 \%$ in $J N K 1^{-/-}$mouse lung and by $7 \%$ in $J N K 2 \& 3^{-/-}$lungs (Figure 1D.) Fractional airspace was increased by $14 \%$ in $J N K 1^{-/-}$mouse lung and by $19 \%$ in $J N K 2 \& 3^{-/-}$lungs (Figure 1E). Both $J N K 1^{-/-}$ and $J N K 2 \& 3^{-/-}$mice had $20 \%$ fewer alveolar crests than wild type mice (Figure 1F). The enlarged distal airspaces and reduced numbers of secondary alveolar septal crests in JNK-deficient mice support a role for JNK in alveolar septation.

\section{JNK reduces lung elastin content}

To test whether JNK suppresses lung elastogenesis, elastin was morphometrically quantitated in PND14 wild type, $J N K 1^{-/-}$and $J N K 2 \& 3^{-/-}$elastin-stained lung sections. Grossly, lung elastin content was increased in $J N K 1^{-1-}$ and $J N K 2 \& 3^{-/-}$lungs compared to wild type (Figure 2A-C). As described in methods, we quantitated the number of dense elastin, diffuse elastin, tissue, and 

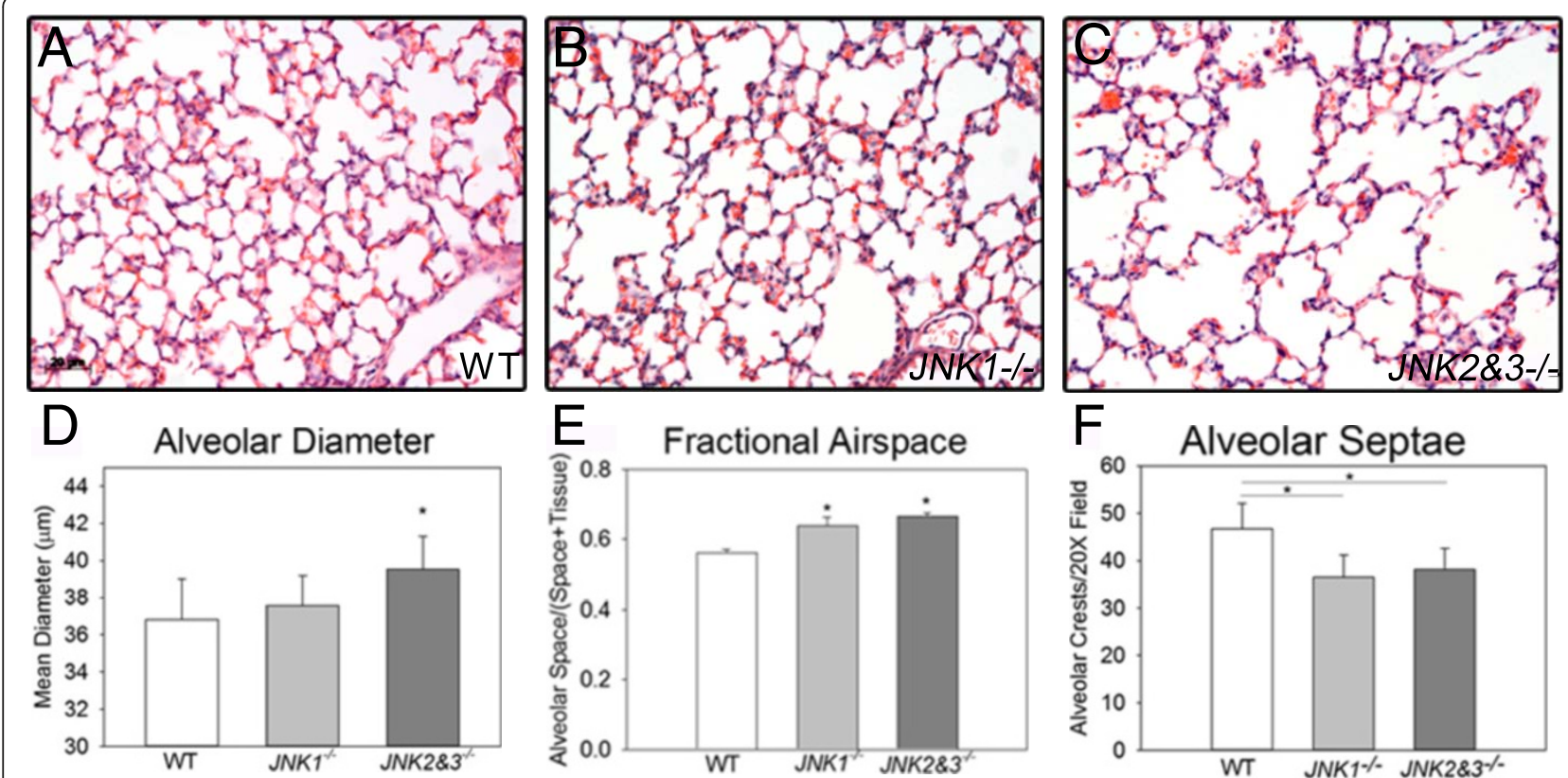

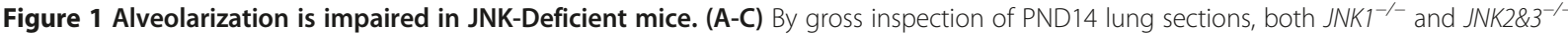

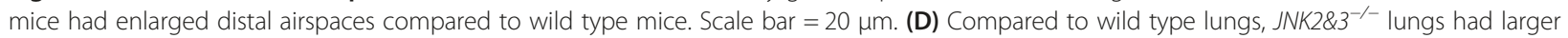
mean alveolar diameter. (E) Compared to wild type lungs, both $J N K 1^{-/-}$and $J N K 2 \& 3^{-/-}$lungs had increased fractional airspace. (F) Both $J N K 1^{-/-}$ and $J N K 2 \& 3^{-1-}$ lungs had decreased numbers of alveolar septa per $20 X$ field. ${ }^{*} \mathrm{p}<0.05$ compared to wild type by one-way ANOVA. Six animals from two different litters were used for analysis except $J \mathrm{NK}^{-1-}$ which contained 3 animals from 3 different litters.

airspace pixels in elastin-stained lung sections from at least three mice from two to three different litters (Figure 2D-G). Wild type lungs contained 7\% more tissue than JNK-deficient lungs (not significant). Normalized to total tissue, $J N K 1^{-1-}$ and $J N K 2 \& 3^{-/-}$lungs had twice the amount of total elastin as wild type lungs (Figure $2 \mathrm{H}$ ). While $J N K 1^{-/-}$lungs had a non-significant increase in dense elastin to total elastin ratio, JNK2\& $3^{-/-}$ lungs had a 1.5-fold increase compared to wild type (Figure 2I). Both $J N K 1^{-/-}$and $J N K 2 \& 3^{-/-}$lungs had 1.7 fold more diffuse elastin than wild type lungs (Figure 2J). By Western blot of PND14 lung homogenates, JNK1 ${ }^{-1-}$ and $J N K 2 \& 3^{-/-}$lung contained 5- and 15 -fold more tropoelastin than wild type lung homogenates respectively (Figure $2 \mathrm{~K}$ ). These data support the hypothesis that JNK suppresses elastogenesis during lung development and plays a role in the localization of elastin fibers to alveolar septal tip.

\section{JNK activity Is increased during postnatal lung development}

To determine whether JNK activity is dynamic over the course of lung development, we immunoprecipitated JNK from lung homogenates and quantitated conversion of c-Jun to phospho-c-Jun. Compared to PN 8 week lung, JNK activity was increased during the saccular and alveolar stages of lung development with the highest JNK activity in PND14 lung homogenates (Figure 3A).
When confirmed in triplicate, JNK activity at PND14 was 11 -fold higher than JNK activity at PN 8 weeks (Figure 3B). By Western blot, phosphorylation of the p54 isoform of JNK was increased in PND14 lung compared to PN 8 week without phosphorylation of the p46 isoform. There were no differences in total JNK protein content (Figure 3C-D). Among the molecules required for elastin fibril assembly are tropoelastin, lysyl oxidase, emilin-1, fibrillin-1, and fibulin-5. To identify an association between JNK activity and elastogenesis, mRNAs of these elastin-associated genes were quantitated at time points between E15.5 and postnatal week eight. The mRNAs of tropoelastin, lysyl oxidase, emilin-1, fibrillin1 , and fibulin-5 were all decreased during late alveolar development (PND14) compared to earlier time points (Figure 3E-F). These data demonstrate an association between decreased transcription of elastin-associated molecules and increased lung JNK activity during later alveolar developent.

\section{JNK activity is localized to pulmonary lipofibroblasts during alveolar development}

We utilized both lineage tracing and immunofluorescent co-localization techniques to determine which cell type was experiencing JNK activation. We first narrowed the range of possible cell types by staining for pJNK in PND5 mesenchymal cell lineage traced lung sections. pJNK was present in a subset of mesenchymal cells and 


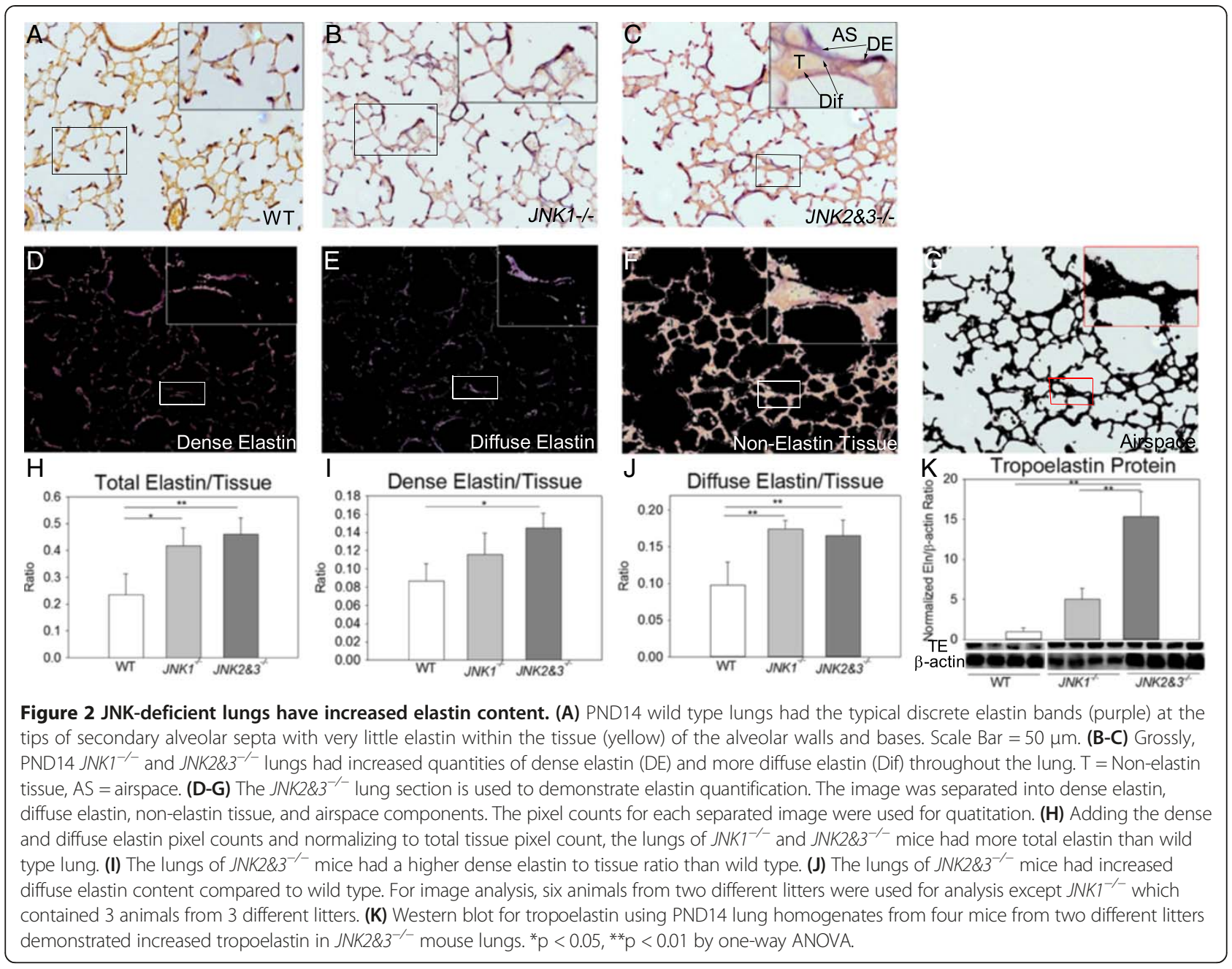

was not activated in epithelial or hematopoietic cells (Figure 4A-E). Since pulmonary fibroblasts, endothelial cells, and pericytes are all derived from the lung mesenchyme, we performed immunostaining for desmin which is expressed in fibroblasts but not the other mesenchymal cell types. pJNK was present only desmin-positive cells (Figure 4F). Pulmonary fibroblasts can grossly be separated into the more fibrogenic myofibroblasts and less fibrogenic lipofibroblasts. Myofibroblasts express higher levels of $\alpha$-smooth muscle actin ( $\alpha$ SMA). pJNK was not located within $\alpha$ SMA positive cells (Figure $4 G$ ). Lipofibroblasts contain increased numbers of lipid droplets, produce less tropoelastin [31,33], and are typically located in alveolar corners [34]. In oil-red-o stained sections, pJNK localized to lipofibroblasts and not to oil-red-o positive macrophages in airways (Figure $4 \mathrm{H}$ ). These data demonstrate that JNK activity during later alveolar development is restricted to pulmonary lipofibroblasts.

\section{JNK suppresses pulmonary fibroblast elastogenesis}

Since JNK suppresses elastogenesis in the aorta [20] and other MAP kinases regulate pulmonary fibroblast elastin synthesis [17], we tested whether JNK suppresses pulmonary fibroblast elastogenesis. Primary PND14 lung fibroblasts were cultured from wild-type, $J N K 1^{-/-}$, and $J N K 2 \& 3^{-/-}$mice. $J N K 1^{-/-}$and JNK $2 \& 3^{-/-}$lung fibroblasts contained 8,000-fold more tropoelastin mRNA than wild type (Figure 5A), and the media from $J N K 1^{-/-}$ and $J N K 2 \& 3^{-/-}$lung fibroblast cultures contained $50 \%$ more soluble tropoelastin (Figure 5B). All three cell types were $50 \%$ confluent at seeding and $100 \%$ confluent at collection. To test whether JNK1 and JNK2 independently suppressed pulmonary fibroblast elastogenesis, JNK1 alleles were deleted from $J N K 2 \& 3^{-/-}$lung fibroblasts using a cre-expressing adenovirus (Ad-Cre). JNK1 mRNA was reduced 35-fold in Ad-Cre infected fibroblasts compared to control (Ad-GFP) (Figure 5C) and $>90 \%$ of Ad-GFP exposed fibroblasts expressed GFP. Deletion of JNK1 

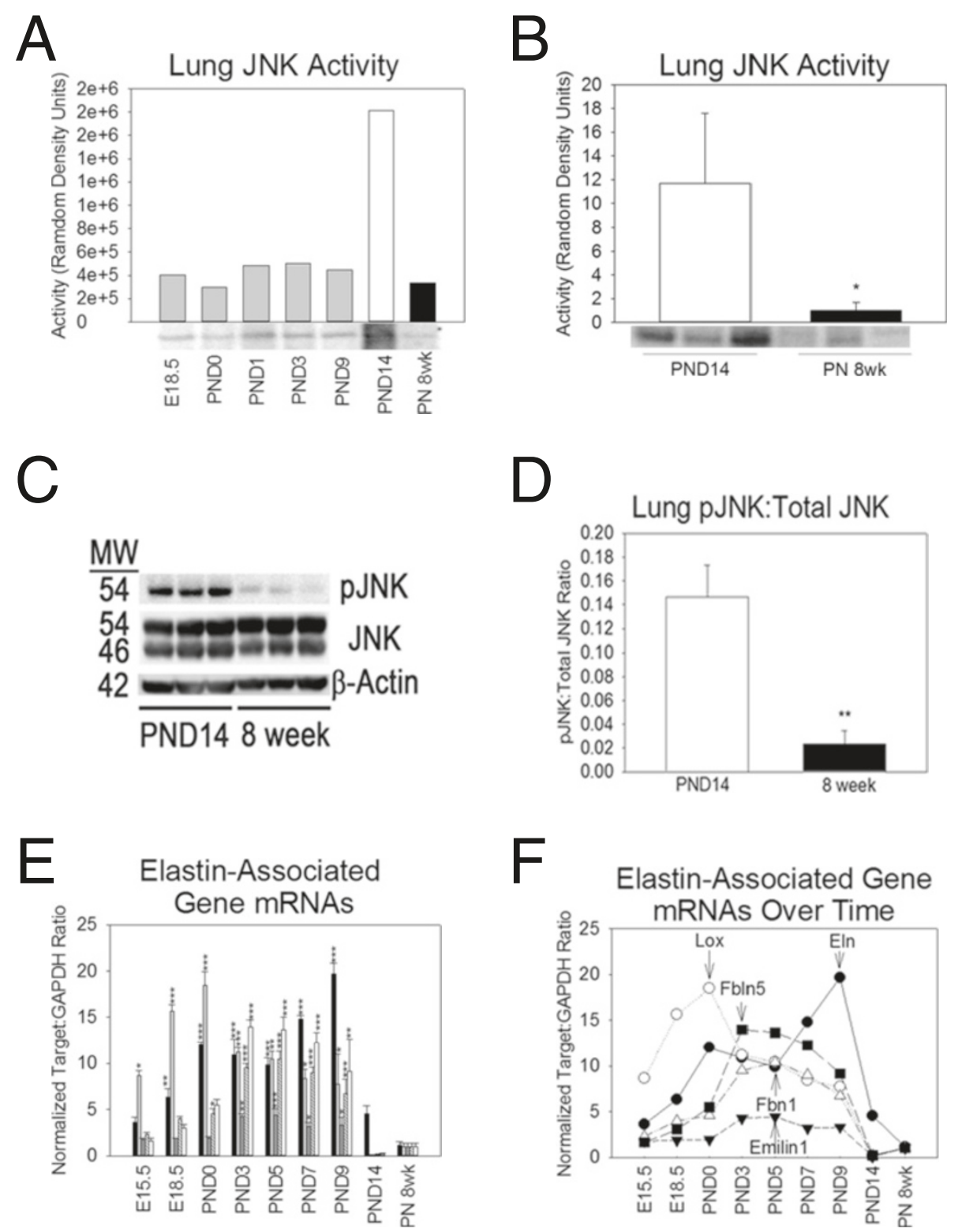

Figure 3 JNK activation and elastogenesis are inversely correlated. (A) By JNK pull down assay, JNK activity (C-Jun phosphorylation) was increased during saccular (PND1\&3) and alveolar (PND9\&14) stages of lung development with activity at PND14 being highest. (B) Comparing the lung JNK activity of mice from different litters, PND14 lungs had 11-fold higher JNK activity than PN 8 week lungs. (C) JNK phosphorylation was assessed by Western blot of lung homogenates from a different set of PND14 and PN 8 week mice (three mice from two different litters). PND14 mice had increased phosphorylation of the p54 JNK isoform but not the p46 isoform. No age-dependent difference in JNK isoform abundance was observed. (D) Western blot densitometry demonstrated a 7-fold increase in pJNK to total JNK ratio in PND14 lung homogenate compared to PN 8 week. (E) Tropoelastin (black bars), lysyl oxidase (gray bars), emilin-1 (dark gray bars), fibrillin-1 (dashed white bars), and fibulin-5 (white bars) mRNAs are all decreased in PND14 and PN 8 week lung compared to time points during saccular and early alveolar lung development (PND0-PND9). RNA is from lungs of three mice from at least two different litters. The noted statistical comparisons are to PN 8 weeks only but analyzed by one-way ANOVA. (F) A line plot of these same data demonstrates that despite different ages of peak mRNA concentration (denoted by arrows) the mRNA concentration of all five elastogenic genes decreases significantly at PND14. ${ }^{*} p<0.05,{ }^{* *} p<0.01,{ }^{* * *} p<0.001$ by one-way ANOVA (>2 groups) or by two tail Student $t$-test (2 groups).

increased tropoelastin mRNA three-fold (Figure 5D). To test whether JNK activation suppresses pulmonary fibroblast elastogenesis, we used the JNK activator anisomycin. Anisomycin reduced PND14 wild-type fibroblast tropoelastin mRNA in a dose-dependent manner that was not statistically significant (Figure 5E). Since epithelial or endothelial cell contamination of primary fibroblast cultures could account for the observed 


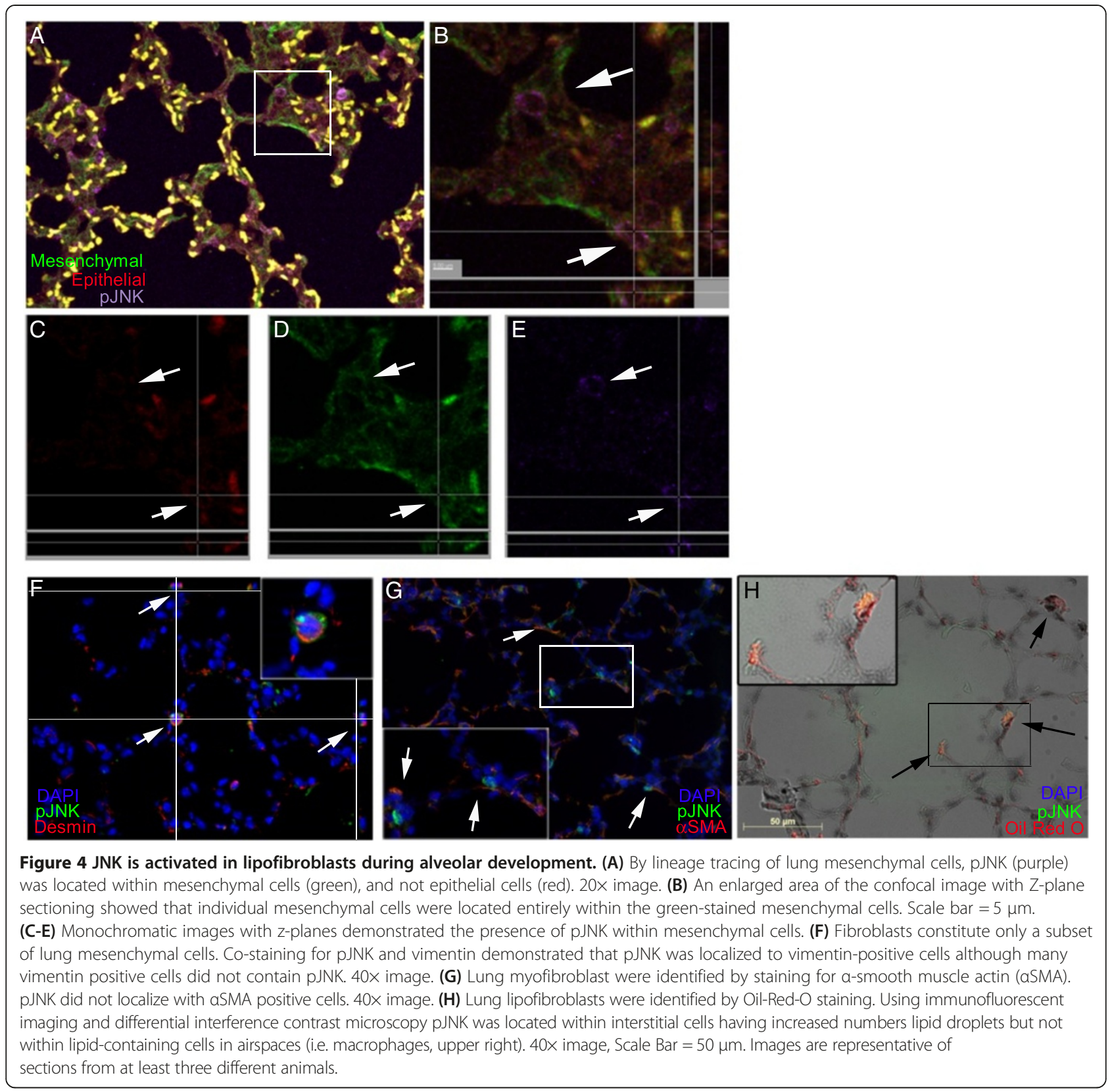

differences, we quantitated surfactant protein B and CD31 (Pecam1) mRNA content. Neither marker could be detected in wild type fibroblast culture but the markers were abundant in wild type lung demonstrating that fibroblasts cultures did not have significant epithelial or endothelial cell contamination. Since JNK3 is not expressed in lung [22], these in vitro data demonstrate that JNK1 and JNK2 independently suppress pulmonary fibroblast elastogenesis.

\section{JNK suppresses mRNAs of elastin-associated genes}

Since the mRNAs of many elastin-associated molecules decreased in association with increased lung JNK activity, and since JNK suppressed elastogenesis in vitro, we tested whether JNK-deficient lungs had increased mRNA levels of elastin-associated molecules. JNK1 $1^{-/-}$ and $J N K 2 \& 3^{-/-}$lungs contained 2,000-fold more emilin1 (Figure 6A) and 700-fold more fibrillin-1 (Figure 6B) than wild type lungs. $J N K 1^{-1-}$ lungs contained 10 -fold and $J N K 2 \& 3^{-/-} 60$-fold more fibulin-5 (Figure $6 \mathrm{C}$ ), but differences in quantities of lysyl oxidase mRNA were small (Figure 6D). JNK1 $1^{-1-}$ and $J N K 2 \& 3^{-/-}$lung contained 6-8 fold more tropoelastin mRNA than wild type (Figure 6E). To confirm that JNK-deficiency itself and not a secondary cell signaling alteration (such as altered mechanical strain or differences in matrix stiffness) was 


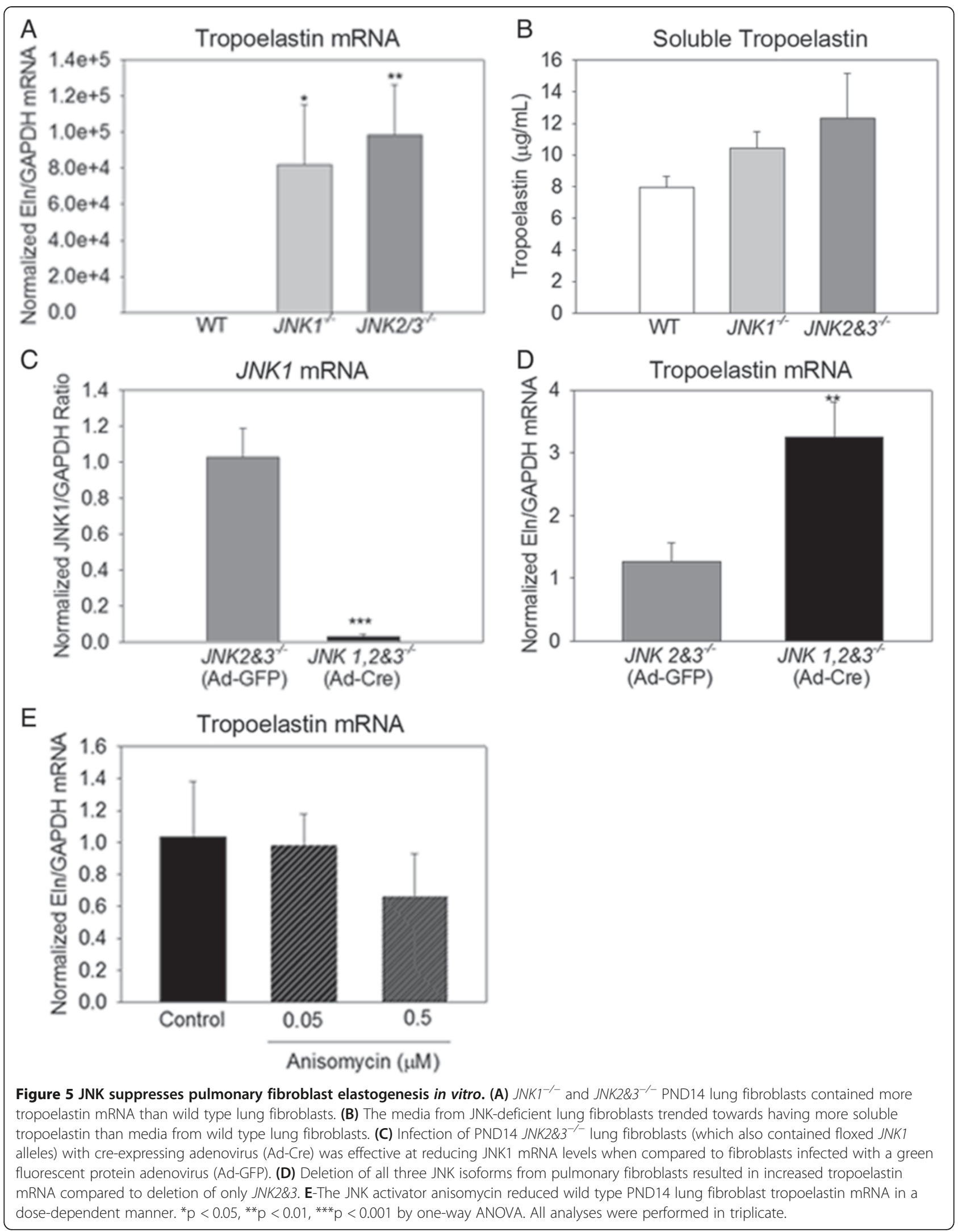




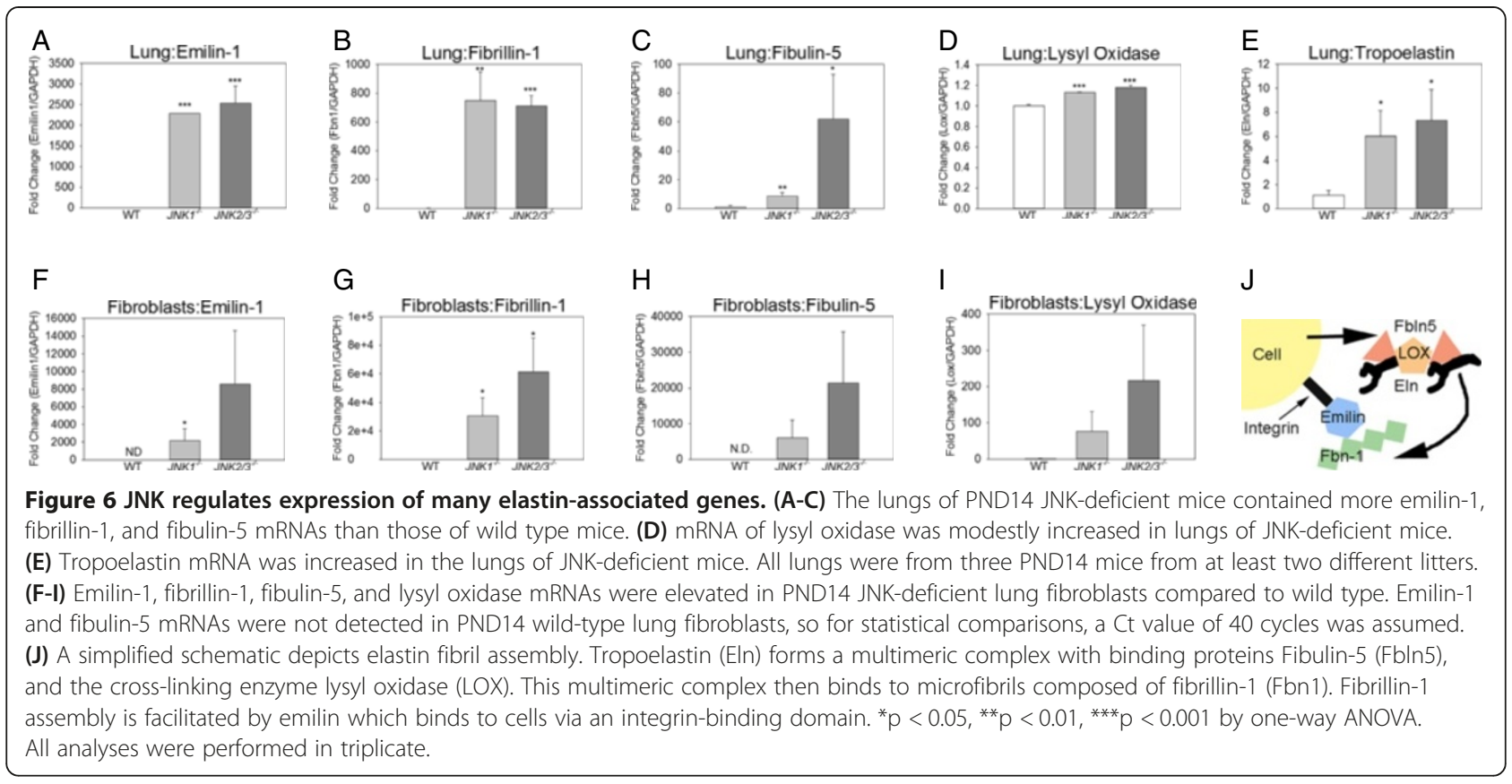

responsible for these differences in elastin-related gene mRNA content, we quantitated these same mRNAs in primary lung fibroblasts. Neither emilin-1 nor fibulin-5 mRNA was detected in wild-type lung fibroblasts but it was present in both $J N K 1^{-/-}$and $J N K 2 \& 3^{-/-}$lung fibroblasts (Figure 6F\&H). Fibrillin-1 mRNA was detected in wild type lung fibroblasts, but mRNA content was several thousand-fold higher in JNK-deficient lung fibroblasts (Figure 6G). Lysyl oxidase mRNA was not statistically different between wild type and JNK-deficient lung fibroblasts (Figure 6I). Tropoelastin mRNA was assessed previously. A schematic depicting how emilin-1, fibrillin-1, fibulin-5, lysyl oxidase, and tropoelastin interact to form elastin fibers is shown in Figure 6J. These data demonstrate that JNK regulates many of the genes involved in elastin microfibril formation and support the concept that JNK suppresses the pulmonary fibroblast elastogenic program.

\section{Discussion}

For the first time, we have demonstrated a role for JNK in suppressing the elastogenic program during the alveolar stage of lung development. JNK activity increased during late alveolar development, and this increased JNK activity was negatively correlated with elastogenic gene mRNAs. JNK-deficiency increased lung elastin content and impaired alveolar septation. Both JNK-deficient lung and lung fibroblasts had increased mRNA levels of elastin-associated genes. Deletion of both JNK1 and JNK2 increased tropoelastin mRNA to a greater extent than either individually demonstrating that both isoforms independently regulate elastogenesis. Our observations are consistent with previous reports demonstrating JNK regulation of elastogenesis in the aorta [20] and AP-1 mediated suppression of tropoelastin expression [35]. Phosphorylated c-jun, the readout of the JNK luciferase assay, is a component of the AP-1 transcriptional complex.

We utilized two methods to assess JNK activation during lung development. In addition to comparison of total JNK to phosphorylated JNK, we utilized a pull-down technique developed and validated at the University of California at San Diego in a variety of cell lines with increasing exposures to ultraviolet irradiation-a well described activator of JNK signaling [32]. This technique quantitates JNK phosphorylation of c-Jun. A drawback of this assay is the absence of a loading control which likely accounts for the increased variability in this assay compared to Western blot. Nonetheless, by both Western blot and JNK pull-down, we demonstrated a negative association between lung JNK activity and elastogenic mRNAs.

We have provided strong in vitro and in vivo loss of function data to support a role for JNK in regulating the elastogenic program in the lung. When assessed in isolation, these effects are clear and strong. However, multiple dynamic processes regulate elastogenesis which likely accounts for larger in vitro than in vivo effects. JNK activity was reduced in adult lung compared to developing lung. This discrepancy is likely due to reduced transcriptional activation of the elastogenic program in the adult compared to the juvenile. Conceptually, JNK activity may serve as a brake slowing elastogenesis during alveolarization which turns off when the elastogenic program is no longer activated. The localization of pJNK 
to pulmonary lipofibroblasts is consistent with their reduced elastogenic and fibrogenic potential [31]; although, the fact that lipofibroblasts increase in number at an earlier age than JNK activity levels increase [36] makes a direct relationship between the two unlikely. The mechanism of how JNK suppresses elastogenesis needs to be determined in future studies.

The necessity of elastin band formation for secondary alveolar septation is well-established $[4,16]$. Both elastin [9] and the lysyl oxidase [37] content are increased in infants with chronic lung disease of prematurity. In mice, elastin haploinsufficiency leads to emphysematous changes [6], and Mammoto et al. recently demonstrated that reduced matrix stiffness also impairs alveolarization [38]. Our data and these previously published data demonstrate that alveolar growth requires both a low compliance elastin band and high compliance alveolar walls. Therapies aimed at restoring alveolar growth should seek to normalize this relationship.

In conclusion, we report that JNK suppresses pulmonary fibroblast elastogenesis during the alveolar stage of lung development plays a role in alveolar septation.

\section{Competing interests}

The authors declare that they have no competing interests.

\section{Authors' contributions}

SL aided in project conceptualization, designed experiments, and performed experiments. HP designed morphometric programs and optimized programs for elastin quantitation. SY performed morphometry and other experiments. BV conceptualized the project, designed experiments, and analyzed data.

\section{Acknowledgements}

We would like to thank Patrick Lahni of the Division of Critical Care Medicine at Cincinnati Children's Hospital Medical Center for his assistance with JNK pull down experiments.

This project was supported with grant support from the National Institutes of Health: K12 HD28827.

\section{Author details}

${ }^{1}$ Cincinnati Children's Hospital Research Foundation, Cincinnati, OH, USA. ${ }^{2}$ Department of Biomedical Engineering, Boston University, Boston, MA, USA. ${ }^{3}$ University of Cincinnati School of Medicine, Cincinnati, OH, USA. ${ }^{4}$ Cincinnati Children's Hospital Medical Center, 3333 Burnet Ave., MLC 2005, Cincinnati, $\mathrm{OH} 45229$, USA.

Received: 18 November 2013 Accepted: 7 March 2014

Published: 25 March 2014

\section{References}

1. Narayanan M, Beardsmore CS, Owers-Bradley J, Dogaru CM, Mada M, Ball I, Garipov RR, Kuehni CE, Spycher BD, Silverman M: Catch-up alveolarization in Ex-preterm children. Evidence from (3)He magnetic resonance. Am J Respir Crit Care Med 2013, 187:1104-1109.

2. Balinotti JE, Tiller CJ, Llapur CJ, Jones MH, Kimmel RN, Coates CE, Katz BP Nguyen JT, Tepper RS: Growth of the lung parenchyma early in life. Am J Respir Crit Care Med 2009, 179:134-137.

3. Wood JP, Kolassa JE, McBride JT: Changes in alveolar septal border lengths with postnatal lung growth. Am J Physiol 1998, 275:L1157-L1163.

4. Mariani TJ, Sandefur S, Pierce RA: Elastin in lung development. Exp Lung Res 1997, 23:131-145.

5. Willet KE, McMenamin P, Pinkerton KE, Ikegami M, Jobe AH, Gurrin L, Sly PD: Lung morphometry and collagen and elastin content: changes during normal development and after prenatal hormone exposure in sheep. Pediatr Res 1999, 45:615-625.

6. Wendel DP, Taylor DG, Albertine KH, Keating MT, Li DY: Impaired distal airway development in mice lacking elastin. Am J Respir Cell Mol Biol 2000, 23:320-326

7. Eberth JF, Popovic N, Gresham VC, Wilson E, Humphrey JD: Time course of carotid artery growth and remodeling in response to altered pulsatility. Am J Physiol Heart Circ Physiol 2010, 299:H1875-H1883.

8. Hamakawa H, Bartolak-Suki E, Parameswaran H, Majumdar A, Lutchen KR, Suki B: Structure-function relations in an elastase-induced mouse model of emphysema. Am J Respir Cell Mol Biol 2011, 45:517-524.

9. Thibeault DW, Mabry SM, Ekekezie II, Truog WE: Lung elastic tissue maturation and perturbations during the evolution of chronic lung disease. Pediatrics 2000, 106:1452-1459.

10. Nicola T, Hagood JS, James ML, Macewen MW, Williams TA, Hewitt MM, Schwiebert L, Bulger A, Oparil S, Chen YF, Ambalavanan N: Loss of Thy-1 inhibits alveolar development in the newborn mouse lung. Am J Physiol Lung Cell Mol Physiol 2009, 296:L738-L750

11. Bruce MC, Pawlowski R, Tomashefski JF Jr: Changes in lung elastic fiber structure and concentration associated with hyperoxic exposure in the developing rat lung. Am Rev Respir Dis 1989, 140:1067-1074.

12. Massaro GD, Massaro D: Postnatal treatment with retinoic acid increases the number of pulmonary alveoli in rats. Am J Physiol 1996, 270:L305-L310.

13. McGowan SE, Jackson SK, Olson PJ, Parekh T, Gold LI: Exogenous and endogenous transforming growth factors-beta influence elastin gene expression in cultured lung fibroblasts. Am J Respir Cell Mol Biol 1997, 17:25-35.

14. Brettell LM, McGowan SE: Basic fibroblast growth factor decreases elastin production by neonatal rat lung fibroblasts. Am J Respir Cell Mol Biol 1994, 10:306-315

15. Yang S, Nugent MA, Panchenko MP: EGF antagonizes TGF-betainduced tropoelastin expression in lung fibroblasts via stabilization of Smad corepressor TGIF. Am J Physiol Lung Cell Mol Physiol 2008, 295:L143-L151.

16. Srisuma S, Bhattacharya S, Simon DM, Solleti SK, Tyagi S, Starcher B, Mariani TJ: Fibroblast growth factor receptors control epithelial-mesenchymal interactions necessary for alveolar elastogenesis. Am J Respir Crit Care Med 2010, 181:838-850.

17. Sproul EP, Argraves WS: A cytokine axis regulates elastin formation and degradation. Matrix Biol 2013, 32:86-94

18. Campbell E, Pierce J, Endicott S, Shapiro S: Evaluation of extracellular matrix turnover. Methods and results for normal human lung parenchymal elastin. Chest 1991, 99:49S.

19. Lepparanta O, Sens C, Salmenkivi K, Kinnula VL, Keski-Oja J, Myllarniemi M, Koli K: Regulation of TGF-beta storage and activation in the human idiopathic pulmonary fibrosis lung. Cell Tissue Res 2012, 348:491-503.

20. Yoshimura K, Aoki H, Ikeda Y, Fujii K, Akiyama N, Furutani A, Hoshii Y, Tanaka N, Ricci R, Ishihara T, Esato K, Hamano K, Matsuzaki M: Regression of abdominal aortic aneurysm by inhibition of c-Jun $\mathrm{N}$-terminal kinase. Nat Med 2005, 11:1330-1338.

21. Das M, Jiang F, Sluss HK, Zhang C, Shokat KM, Flavell RA, Davis RJ: Suppression of p53-dependent senescence by the JNK signal transduction pathway. Proc Natl Acad Sci USA 2007, 104:15759-15764.

22. Davis RJ: Signal transduction by the JNK group of MAP kinases. Cell 2000, 103:239-252.

23. Cornett B, Snowball J, Varisco BM, Lang R, Whitsett J, Sinner D: Wntless is required for peripheral lung differentiation and pulmonary vascular development. Dev Biol 2013, 379:38-52

24. Hilgendorff A, Parai K, Ertsey R, Jain N, Navarro EF, Peterson JL, Tamosiuniene R, Nicolls MR, Starcher BC, Rabinovitch M, Bland RD: Inhibiting lung elastase activity enables lung growth in mechanically ventilated newborn mice. Am J Respir Crit Care Med 2011, 184:537-546.

25. Jacob RE, Carson JP, Gideon KM, Amidan BG, Smith CL, Lee KM Comparison of two quantitative methods of discerning airspace enlargement in smoke-exposed mice. PLoS One 2009, 4:e6670.

26. Parameswaran H, Majumdar A, Ito S, Alencar AM, Suki B: Quantitative characterization of airspace enlargement in emphysema. J Appl Physiol 2006, 100:186-193. 
27. Wilson AA, Murphy GJ, Hamakawa H, Kwok LW, Srinivasan S, Hovav AH, Mulligan RC, Amar S, Suki B, Kotton DN: Amelioration of emphysema in mice through lentiviral transduction of long-lived pulmonary alveolar macrophages. J Clin Invest 2010, 120:379-389.

28. Chen ZH, Lam HC, Jin Y, Kim HP, Cao J, Lee SJ, Ifedigbo E, Parameswaran H, Ryter SW, Choi AM: Autophagy protein microtubule-associated protein 1 light chain-3B (LC3B) activates extrinsic apoptosis during cigarette smoke-induced emphysema. Proc Natl Acad Sci USA 2010, 107:18880-18885

29. Perl AK, Gale E: FGF signaling is required for myofibroblast differentiation during alveolar regeneration. Am J Physiol Lung Cell Mol Physiol 2009, 297:L299-L308.

30. Whitsett JA, Ross G, Weaver T, Rice W, Dion C, Hull W: Glycosylation and secretion of surfactant-associated glycoprotein A. J Biol Chem 1985, 260:15273-15279.

31. Varisco BM, Ambalavanan N, Whitsett JA, Hagood JS: Thy-1 signals through PPARgamma to promote lipofibroblast differentiation in the developing lung. Am J Respir Cell Mol Biol 2012, 46:765-772.

32. Hibi M, Lin A, Smeal T, Minden A, Karin M: Identification of an oncoprotein- and UV-responsive protein kinase that binds and potentiates the c-Jun activation domain. Genes Dev 1993, 7:2135-2148.

33. Sanders YY, Kumbla P, Hagood JS: Enhanced myofibroblastic differentiation and survival in Thy-1(-) lung fibroblasts. Am J Respir Cell Mol Biol 2007, 36:226-235.

34. McGowan SE, Torday JS: The pulmonary lipofibroblast (lipid interstitial cell) and its contributions to alveolar development. Annu Rev Physiol 1997, 59:43-62.

35. Kahari VM, Chen YQ, Bashir MM, Rosenbloom J, Uitto J: Tumor necrosis factor-alpha down-regulates human elastin gene expression. Evidence for the role of AP-1 in the suppression of promoter activity. J Biol Chem 1992, 267:26134-26141.

36. Rehan V, Torday J: Hyperoxia augments pulmonary lipofibroblastto-myofibroblast transdifferentiation. Cell Biochem Biophys 2003, 38:239-250.

37. Kumarasamy A, Schmitt I, Nave AH, Reiss I, van der Horst I, Dony E, Roberts JD Jr, de Krijger RR, Tibboel D, Seeger W, Schermuly RT, Eickelberg O, Morty RE: Lysyl oxidase activity is dysregulated during impaired alveolarization of mouse and human lungs. Am J Respir Crit Care Med 2009, 180:1239-1252.

38. Mammoto $T$, Jiang E, Jiang $A$, Mammoto A: Extracellular matrix structure and tissue stiffness control postnatal lung development through the lipoprotein receptor-related protein 5/Tie2 signaling system. Am J Respir Cell Mol Biol 2013, 49:1009-1018.

doi:10.1186/1465-9921-15-34

Cite this article as: Liu et al: JNK suppresses pulmonary fibroblast elastogenesis during alveolar development. Respiratory Research 2014 15:34.

\section{Submit your next manuscript to BioMed Central and take full advantage of:}

- Convenient online submission

- Thorough peer review

- No space constraints or color figure charges

- Immediate publication on acceptance

- Inclusion in PubMed, CAS, Scopus and Google Scholar

- Research which is freely available for redistribution 